\title{
Latest results on hadronic resonance production with ALICE at the LHC
}

\section{Dukhishyam Mallick for the ALICE Collaboation ${ }^{a, *}$}

${ }^{a}$ National Institute of Science Education and Research, HBNI, Jatni, India

E-mail: dukhishyam.mallick@cern.ch

Resonances with very short lifetimes can be used to probe the rescattering and regeneration processes in the hadronic phase of the system produced after a high-energy collisions. These processes are studied by measuring resonance yields as a function of the system size and collision energy and comparing them to model calculations with and without the hadronic cascades. We present measurements of transverse momentum spectra, integrated yields $(\mathrm{d} N / \mathrm{d} y)$, mean transverse momenta $\left(\left\langle p_{\mathrm{T}}\right\rangle\right)$, and angular distributions for light flavor hadronic resonances in $\mathrm{pp}, \mathrm{p}-\mathrm{Pb}$, $\mathrm{Xe}-\mathrm{Xe}$, and $\mathrm{Pb}-\mathrm{Pb}$ collisions at LHC energies. The $\left\langle p_{\mathrm{T}}\right\rangle$ of resonances as a function of event multiplicity in central $\mathrm{Pb}-\mathrm{Pb}$ collisions follow the same mass ordering as for other hadrons, expected from the hydrodynamic expansion of the system. At high $p_{\mathrm{T}}(>8 \mathrm{GeV} / c)$, nuclear modification factor $\left(R_{\mathrm{AA}}\right)$ of light flavor hadrons in central $\mathrm{Pb}-\mathrm{Pb}$ collisions shows strong suppression, whereas the nuclear modification factor in $\mathrm{p}-\mathrm{Pb}$ collisions, known as $R_{\mathrm{pPb}}$ is consistent with unity. This parton energy loss effect is independent of the particle species. Further, in non-central heavy-ion collisions, the vector mesons can be polarized due to spin-orbital-angular-momentum interaction or hadronization from polarized quarks due to their significant initial angular momentum. Recent measurements of spin alignment for $\mathrm{K}^{0}$ and $\phi$ mesons produced at midrapidity in $\mathrm{pp}$ and $\mathrm{Pb}-\mathrm{Pb}$ collisions are discussed.

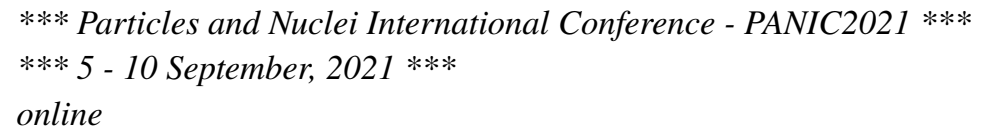

${ }^{*}$ Speaker 


\section{Itroduction}

The study of hadronic-resonance production serves as a unique tool to understand the properties of the hadronic medium created in high energy collisions. Since they have lifetimes comparable to the hadronic phase, rescattering and regeneration processes may affect the measured yields. These processes also modify the resonance momentum distributions. Recent measurements in pp and $\mathrm{p}-\mathrm{Pb}$ collisions as a function of event multiplicity have shown similar features [1] to those seen in heavy-ion collisions [2]. A systematic measurements related to resonances may enable us to further investigate the dynamics of the hadronic phase and study the hadronic phase's lifetime. The nuclear modification factor for resonances helps in understanding parton energy loss in a hot and dense medium, distinguishing between the initial and final state effects. The large angular momentum $\mathrm{O}\left(10^{7}\right) \hbar$ and magnetic field $\mathrm{O}\left(10^{15}\right) \mathrm{T}$ are created in non-central heavy-ion collisions at the LHC energies [3]. Spin alignment of vector mesons ( significant initial angular momentum of the system [4] or during the process of hadronization [5]. The initial magnetic field is short-lived, whereas the angular momentum is a conserved quantity felt throughout the evolution of a system formed in heavy-ion collisions. The angular distribution of decay daughters of vector mesons is an excellent experimental candidate to probe these initial conditions of heavy-ion collisions. The angular distribution of decay products of vector mesons is described by the equation [6]

$$
\frac{d N}{d \cos \theta^{*}}=N_{0} \times\left[\left(1-\rho_{00}\right)+\left(3 \rho_{00}-1\right) \cos ^{2} \theta^{*}\right]
$$

where $\mathrm{N}_{0}$ is the normalization constant, $\rho_{00}$ is the probability that vector mesons in zeroth spin state and $\theta^{*}$ is the angle between the polarization axis and the momentum direction of a daughter particle in the rest frame of the vector meson.

\section{Analysis details}

A detailed description of the ALICE detector setup and its performance is discussed in ref. [7]. The sub-detectors relevant for these studies are the Time Projection Chamber (TPC), the Time-ofFlight detector (TOF), the Inner Tracking System (ITS), covering pseudorapidity window of $|\eta|<$ 0.9 , and the V0A $(2.8<\eta<5.1)$ and V0C $(3.7<\eta<1.7)$ detectors. The ITS and TPC are used for charged-particle tracking and primary vertex reconstruction, whereas particle identification is done by measuring specific energy loss in the detector gas of TPC and the time-of-flight information using TOF. The V0 detectors are used for triggering and estimation of multiplicity/centrality at forward rapidities. The measurements of hadronic resonance production are performed at midrapidity $(|y|<$ 0.5 ) in $\mathrm{pp}, \mathrm{Xe}-\mathrm{Xe}$, and $\mathrm{Pb}-\mathrm{Pb}$ collisions and in $-0.5<y<0.0$ for $\mathrm{p}-\mathrm{Pb}$ collisions as a function of the charged-particle multiplicity. The invariant-mass method is used to reconstruct resonances from their decay products. The combinatorial background has been estimated using event mixing or like-sign method depending on the analysis. For example, the invariant mass distributions for the $\mathrm{K}^{* 0}(\phi)$ signal are fitted with a Breit-Wigner (Voigtian: convolution of Breit-Wigner and Gaussian distributions) function and a second order polynomial that describes the residual background, in order to extract the yields. The raw yields are extracted from the signal distribution after the subtraction of the combinatorial background and further corrected for reconstruction efficiency and 
acceptance and branching ratio. For spin alignment studies, extracted yields are corrected for the reconstruction efficiency and acceptance in each $\cos \theta^{*}$ and $p_{\mathrm{T}}$ bin. The corrected $\mathrm{d} N / \mathrm{d} \cos \theta^{*}$ distributions are fitted with the functional form given in the eqn.1 to determine $\rho_{00}$ for each $p_{\mathrm{T}}$ bins [3].

\section{Results and discussions}

The yield of $\mathrm{K}^{* 0}$ scaled by $\left\langle\mathrm{d} N_{\mathrm{ch}} / \mathrm{d} \eta\right\rangle_{|\eta|<0.5}$ as a function of $\left\langle\mathrm{d} N_{\mathrm{ch}} / \mathrm{d} \eta\right\rangle_{|\eta|<0.5}$ in pp collisions at $\sqrt{s}=7$ and $13 \mathrm{TeV}$, and in $\mathrm{p}-\mathrm{Pb}$ collisions at $\sqrt{s_{\mathrm{NN}}}=5.02$ and $8.16 \mathrm{TeV}$ is shown in the left panel of Fig. 1. The scaled integrated yields are similar and consistent with each other within uncertainties at given $\left\langle\mathrm{d} N_{\mathrm{ch}} / \mathrm{d} \eta\right\rangle_{|\eta|<0.5}$, irrespective of the colliding systems and energies. The right panel of Fig. 1 shows $\left\langle p_{\mathrm{T}}\right\rangle$ of resonances $\left(\mathrm{K}^{* 0}\right.$ and $\left.\phi\right)$ along with identified hadrons $\left(\pi^{ \pm}, \mathrm{K}^{ \pm}\right.$and $\left.\mathrm{p}(\overline{\mathrm{p}})\right)$ as a function of $\left\langle\mathrm{d} N_{\mathrm{ch}} / \mathrm{d} \eta\right\rangle_{|\eta|<0.5}$ in $\mathrm{Pb}-\mathrm{Pb}$ collisions at $\sqrt{s_{\mathrm{NN}}}=5.02 \mathrm{TeV}$. The $\left\langle p_{\mathrm{T}}\right\rangle$ increases with $\left\langle\mathrm{d} N_{\mathrm{ch}} / \mathrm{d} \eta\right\rangle_{|\eta|<0.5}$ for all hadrons. It also increases with mass hadrons. A mass ordering for similar mass of hadrons $\left(\mathrm{p}, \mathrm{K}^{* 0}\right.$ and $\left.\phi\right)$ observed at central $\mathrm{Pb}-\mathrm{Pb}$ collisions as expected from hydrodynamics, whereas mass ordering breaks down in peripheral $\mathrm{Pb}-\mathrm{Pb}$ collisions [2] and small colliding systems $[1,9,10]$. Left of the Fig. 2 shows $\mathrm{K}^{* 0} / \mathrm{K}$ and $\phi / \mathrm{K}$ as a function of $\left\langle\mathrm{d} N_{\mathrm{ch}} / \mathrm{d} \eta\right\rangle^{1 / 3}$ in different colliding systems
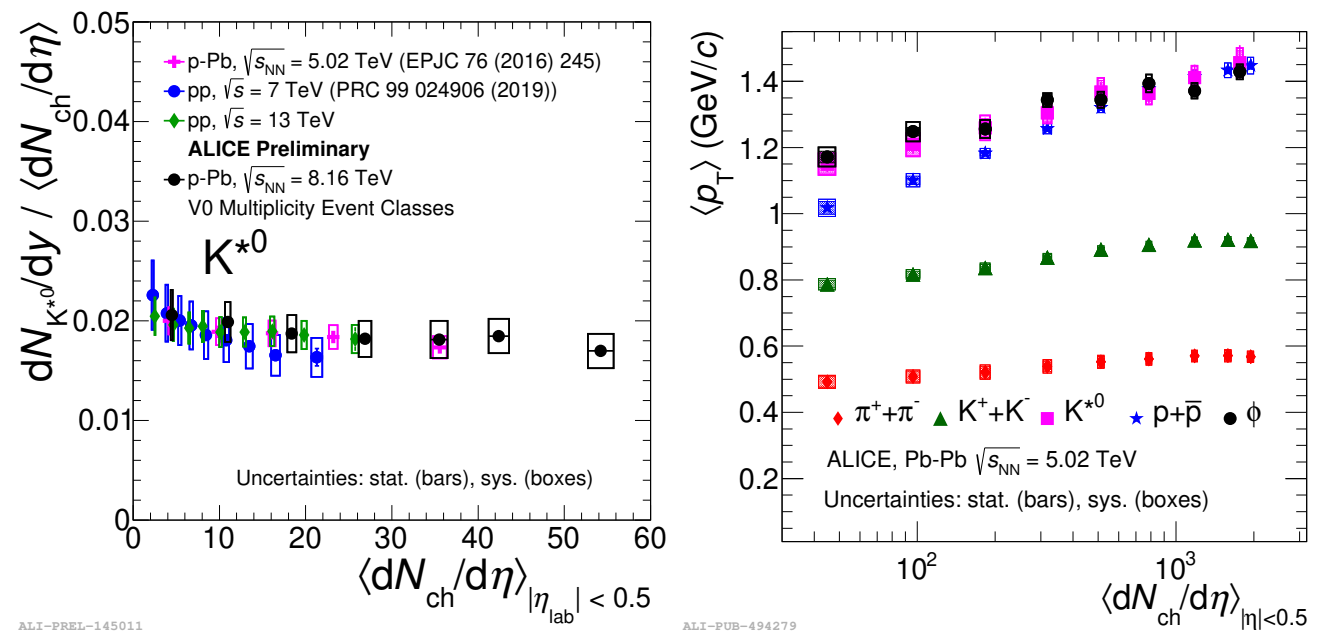

Figure 1: Left: Scaled integrated yield $\left(\mathrm{d} N / \mathrm{d} y /\left\langle\mathrm{d} N_{\mathrm{ch}} / \mathrm{d} \eta\right)\right.$ of $\mathrm{K}^{* 0}$ as a function of charged particle multiplicity $\left(\left\langle\mathrm{d} N_{\mathrm{ch}} / \mathrm{d} \eta\right\rangle_{\left|\eta_{\text {lab }}\right|<0.5}\right)$ in $\mathrm{p}-\mathrm{Pb}$ collisions at $\sqrt{s_{\mathrm{NN}}}=8.16 \mathrm{TeV}$ are compared with pp collisions at $\sqrt{s}=7,13$ $\mathrm{TeV}$, and $\mathrm{p}-\mathrm{Pb}$ collisions at $\sqrt{s_{\mathrm{NN}}}=5.02 \mathrm{TeV}$. Right: Mean transverse momentum of $\mathrm{K}^{* 0}$ and $\phi$ compared with identified hadrons such as $\pi^{ \pm}, \mathrm{K}^{ \pm}, \mathrm{p}(\overline{\mathrm{p}})$ as a function of $\left\langle\mathrm{d} N_{\mathrm{ch}} / \mathrm{d} \eta\right\rangle_{|\eta|<0.5}$ for $\mathrm{Pb}-\mathrm{Pb}$ collisions at $\sqrt{s_{\mathrm{NN}}}$ $=5.02 \mathrm{TeV}$.

and energies measured by the ALICE. A decreasing trend of $\mathrm{K}^{* 0} / \mathrm{K}$ with $\left\langle\mathrm{d} N_{\mathrm{ch}} / \mathrm{d} \eta\right\rangle^{1 / 3}$ is observed from pp to central $\mathrm{Pb}-\mathrm{Pb}$ collisions, whereas $\phi / \mathrm{K}$ ratios are nearly constant across all systems and centrality classes. These results suggest dominance of re-scattering effects over regeneration in the hadronic phase [2]. The lifetime of hadronic phase is estimated from the measured $\mathrm{K}^{* 0} / \mathrm{K}$ ratios of the order $\sim 4-7 \mathrm{fm} / c$ for central $\mathrm{Pb}-\mathrm{Pb}$ collisions at $\sqrt{s_{\mathrm{NN}}}=5.02 \mathrm{TeV}$ [8], by assuming neglisible effects of regeneration processes between chemical to kinematic freeze-out. In the right panel of 
Fig. 2, it is shown the comparison of the nuclear modification factor $\left(R_{\mathrm{pPb}}\right)$ of $\mathrm{K}^{* 0}, \mathrm{~K}^{* \pm}$ and $\phi$ along with $\pi, \mathrm{K}, \mathrm{p}$ at $\sqrt{s_{\mathrm{NN}}}=5.02 \mathrm{TeV}$ in $\mathrm{p}-\mathrm{Pb}$ collisions. At $p_{\mathrm{T}}>8 \mathrm{GeV} / c, R_{\mathrm{pPb}}$ values are consistent within unity whereas deviations from unity are observed for $R_{\mathrm{AA}}$ in $\mathrm{Pb}-\mathrm{Pb}$ collisions [2]. The $R_{\mathrm{pPb}}$ or $R_{\mathrm{AA}}$ of all light flavor particles are consistent within uncertainties at high $p_{\mathrm{T}}$, indicating that these is not flavor dependence in parton energy loss.
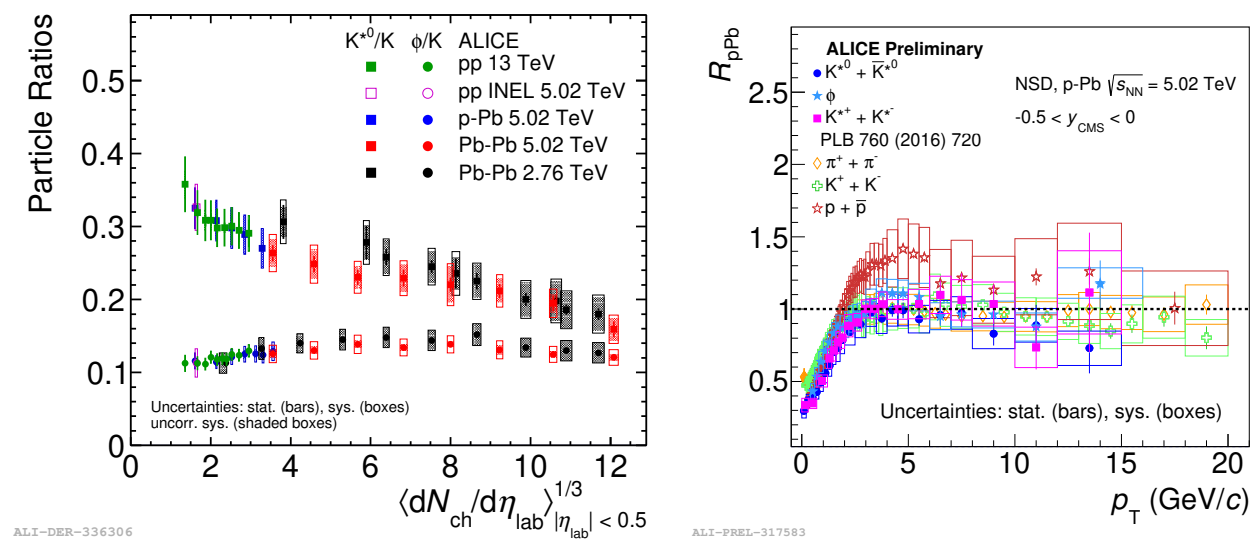

Figure 2: Left: Summary of $p_{\mathrm{T}}$-integrated yield ratios of resonances to their ground state particles $\left(\mathrm{K}^{* 0} / \mathrm{K}\right.$, $\phi / \mathrm{K})$ as a function of $\left\langle\mathrm{d} N_{\mathrm{ch}} / \mathrm{d} \eta\right\rangle^{1 / 3}$ in $\mathrm{pp}, \mathrm{p}-\mathrm{Pb}$ and $\mathrm{Pb}-\mathrm{Pb}$ collisions. Right: Nuclear modification factor of $\mathrm{K}^{* 0}, \mathrm{~K}^{* \pm}$, and $\phi$ as a function of $p_{\mathrm{T}}$, are compared with $\pi, \mathrm{K}$, and $\mathrm{p}$ in $\mathrm{p}-\mathrm{Pb}$ collisions at $\sqrt{s_{\mathrm{NN}}}=5.02 \mathrm{TeV}$.

Figure 3 shows the $\rho_{00}$ as a function of $p_{\mathrm{T}}$ for $\mathrm{K}^{* 0}$ and $\phi$ mesons in $\mathrm{pp}$ and $\mathrm{Pb}-\mathrm{Pb}$ collisions, along with the measurements for $\mathrm{K}_{0}^{s}$ in $\mathrm{Pb}-\mathrm{Pb}$ collisions. In midcentral (10-50\%) $\mathrm{Pb}-\mathrm{Pb}$ collisions, $\rho_{00}$ is below $1 / 3$ for the lowest measured $p_{\mathrm{T}}$ interval and increases to $1 / 3$ within uncertainties for $p_{\mathrm{T}}>2 \mathrm{GeV} / c$. At low $p_{\mathrm{T}}$, the central value of $\rho_{00}$ is smaller for $\mathrm{K}^{* 0}$ than for $\phi$, although the results are compatible within uncertainties. In pp collisions, $\rho_{00}$ is independent of $p_{\mathrm{T}}$ and equal to $1 / 3$ within uncertainties. For the spin zero hadron $\mathrm{K}_{\mathrm{s}}^{0}, \rho_{00}$ is consistent with $1 / 3$ within uncertainties in $\mathrm{Pb}-\mathrm{Pb}$ collisions. The results with random event plane directions are also compatible with no spin alignment for the measured $p_{\mathrm{T}}$ range.

\section{Conclusions}

ALICE has studied several resonances with varying lifetimes (from 1-50 fm/c) in different colliding systems and energies, suggesting that event multiplicity drives the resonance production. The mass ordering in the average transvesre momentum for central $\mathrm{Pb}-\mathrm{Pb}$ collisions breaks down for peripheral $\mathrm{Pb}-\mathrm{Pb}, \mathrm{p}-\mathrm{Pb}$ and $\mathrm{pp}$ collisions. Evidence of rescattering effects is found due to the suppression of the $\mathrm{K}^{* 0} / \mathrm{K}$ ratio in central $\mathrm{Pb}-\mathrm{Pb}$ collisions compared the corresponding value in pp collisions. The $R_{\mathrm{pPb}}$ values show a lack of species dependence and are consistent with unity within the uncertainties for light flavor hadrons at high- $p_{\mathrm{T}}(>8 \mathrm{GeV} / \mathrm{c})$. The spin alignment of vector mesons is observed at low $p_{\mathrm{T}}$ for midcentral collisions at midrapidity in $\mathrm{Pb}-\mathrm{Pb}$ collisions, which is surprisingly larger relative to the polarization measured for hyperon. In the future, precise measurement of energy dependence study of $\rho_{00}$ and the difference of spin alignment of $\mathrm{K}^{* 0}$ and $\mathrm{K}^{* \pm}$ due to their different magnetic moment are possible. Further, the availability of high statistics 


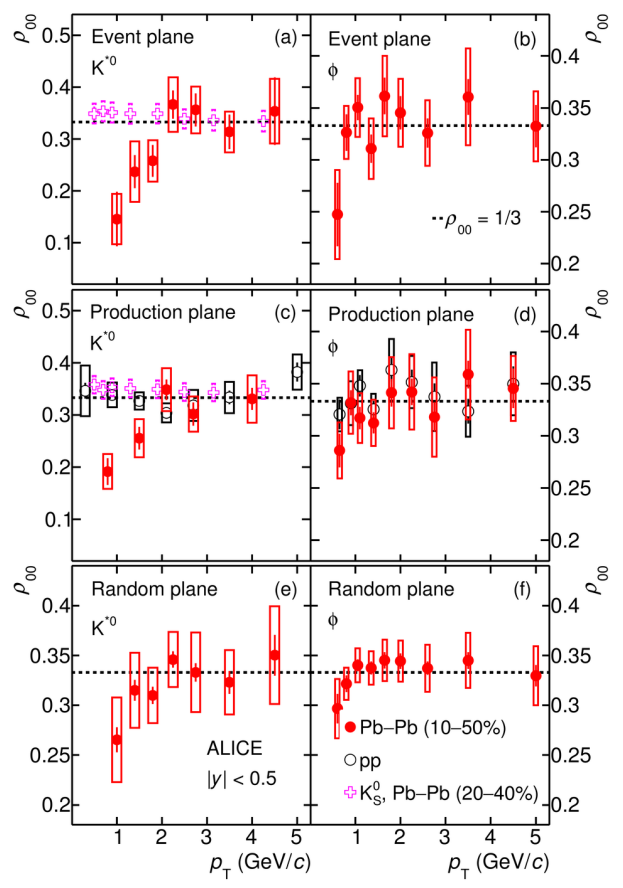

Figure 3: The $\rho_{00}$ as a function of $p_{\mathrm{T}}$ for vector mesons $\left(\mathrm{K}^{* 0}\right.$ and $\left.\phi\right)$ and $K_{0}^{s}$ at midrapidity in $\mathrm{Pb}-\mathrm{Pb}$ collisions at $\sqrt{s_{\mathrm{NN}}}=2.76 \mathrm{TeV}$ and minimum bias pp collisions at $\sqrt{s}=13 \mathrm{TeV}$. Results are shown for spin alignment with respect to event plane (panels a,b), production plane (c,d) and random event plane (e,f) for $\mathrm{K}^{* 0}$ (left column) and $\phi$ (right column). The statistical and systematic uncertainties are shown as bars and boxes, respectively

Run2 and upcoming Run3 data will allow exploring higher mass rare resonances such as $\mathrm{f}_{0}(980)$, $\mathrm{f}_{2}(1525), \Xi(1820)$, etc.

\section{References}

[1] J.Adam et al.[ALICE], Eur. Phys. J. C 76, 245

[2] S.Acharya et al.[ALICE], arXiv:2106.13113

[3] S.Acharya et al.[ALICE], Phys. Rev. Lett. 125, 012301

[4] Phys. Rev. C 77, 024906

[5] Phys. Lett. B 682, 408

[6] Phys. Rev. C 77, 061902

[7] K. Aamodt et al. [ALICE], JINST 3, S08002.

[8] S.Acharya et al.[ALICE], PLB 802, 135225

[9] S.Acharya et al.[ALICE], Phys.Lett.B 807,135501

[10] S.Acharya et al.[ALICE], arXiv:2110.10042 